\title{
Closed Form Solutions of Linear Odes having Elliptic Function Coefficients
}

\author{
Reinhold Burger \\ Symbolic Computation Group \\ School of Computer Science \\ University of Waterloo \\ Waterloo, Ontario, Canada \\ rfburger@scg.uwaterloo.ca
}

\author{
George Labahn \\ Symbolic Computation Group \\ School of Computer Science \\ University of Waterloo \\ Waterloo, Ontario, Canada \\ glabahn@uwaterloo.ca
}

\author{
Mark van Hoeij \\ Department of Mathematics \\ Florida State University \\ Tallahassee, Florida, USA \\ hoeij@math.fsu.edu
}

\begin{abstract}
We consider the problem of finding closed form solutions of linear differential equations having coefficients which are elliptic functions. For second order equations we show how to solve such an ode in terms of doubly periodic functions of the second kind. The method depends on two procedures, the first using a second symmetric power of an ode along with a decision procedure for determining when such equations have elliptic function solutions while the second involves the computation of exponential solutions.
\end{abstract}

\section{Categories and Subject Descriptors}

I.1.2 [Computing Methodologies]: Symbolic and Algebraic Manipulation-Algorithms

\section{General Terms}

Algorithms

\section{Keywords}

Linear odes; elliptic functions; Kovacic's algorithm; decision procedures

\section{INTRODUCTION}

In this paper we consider linear differential equations of the form

$$
a_{n}(x) y^{(n)}(x)+\cdots+a_{1}(x) y^{\prime}(x)+a_{0}(x) y(x)=0
$$

where the coefficients $a_{i}(x)$ are doubly periodic having the same periods. Doubly periodic functions are complex-valued functions having two independent periods, that is, two independent constants $T$ and $T^{\prime}$ such that

$$
f(x+T)=f(x) \text { and } f\left(x+T^{\prime}\right)=f(x) \text { for all } x .
$$

Permission to make digital or hard copies of all or part of this work for personal or classroom use is granted without fee provided that copies are not made or distributed for profit or commercial advantage and that copies bear this notice and the full citation on the first page. To copy otherwise, to republish, to post on servers or to redistribute to lists, requires prior specific permission and/or a fee.

ISSAC'04, July 4-7, 2004, Santander, Spain.

Copyright 2004 ACM 1-58113-827-X/04/0007 ...\$5.00
Classical examples of doubly periodic functions include the Weierstrass $\wp$ and $\wp^{\prime}$ functions, the Jacobi $s n$, $c n$ and $d n$ functions and ratios of Theta functions [1]. We will also require that our coefficients are in fact elliptic functions, that is, that they do not have any essential singularities. We remark that the term doubly periodic is often used when elliptic is really meant, it being assumed that the functions under discussion are also meromorphic.

Linear differential equations with elliptic function coefficients appear historically in many instances (cf. [9]). For example, solving Laplace's equation in three dimensions in ellipsoidal coordinates using the separation of variables method gives the classical Lamé equation

$$
y^{\prime \prime}(x)-(n(n+1) \wp(x)+B) y(x)=0
$$

where $n$ is a positive integer and $B$ is a constant. Additional interesting examples can be found in many texts, see for example Forsyth [9], Halphen [10] and Kamke [14].

There are two classical representations of elliptic functions, the Weierstrass form and the Jacobi form. In either case, we can convert (1) to a linear ode having coefficients from $\mathbb{K}(z, \sqrt{w(z)})$ where $w(z)$ is a polynomial of degree 3 or 4 and $K$ is a field of constants. Using methods from [16, 17] one obtains a decision procedure for determining when (1) has solutions which are elliptic functions.

Picard's theorem $[9,15]$ says that when all solutions of (1) are uniform or path-independent then the ode has solutions that are doubly periodic of the second kind. These functions can be expressed in terms of the Weierstrass Zeta and Sigma functions or the Jacobi Zeta function [1]. In this paper we show how we can find such solutions in the case of all second order linear odes, even when all solutions are not necessarily uniform. In fact we present a complete algorithm for solving reducible second order linear odes with elliptic function coefficients. We do this by finding elliptic function solutions of the second symmetric power of the original ode (in section 3), or else finding exponential solutions of the symmetric product of the equation with its conjugate (in section 4). The methods have been implemented [7] and are available (or will shortly be available) in the Maple computer algebra system. We mention that our method is not the first algorithm for finding such solutions. Indeed the procedures in [17] find all Liouvillian solutions of (1), in both the reducible or irreducible cases. Our contribution is to provide a new method which both efficient and complete for the second order reducible case of (1). 
The remainder of the paper is organized as follows. In the next section we give background information for odes of the form (1). Section 3 gives a method for solving the second order problem in terms of doubly-periodic functions of the second kind when there are elliptic function solutions of an associated third order equation while the following section produces solutions which are doubly-periodic functions of the second kind via finding exponential solutions of an associated ode of fourth order. The paper ends with a conclusion along with topics for future research.

\section{PRELIMINARIES}

In this section we give some well-known facts about elliptic functions, doubly periodic functions of the second kind and linear odes having elliptic functions as coefficients. Additional information and details can be found in the references $[2,3,9,13]$.

\subsection{Elliptic Function Solutions of Arbitrary Order Equations}

There are two classical representations of elliptic functions, the Weierstrass form and the Jacobi form (c.f. [3]). Let $\wp(x)=\wp\left(x ; g_{2}, g_{3}\right)$ denote the Weierstrass $\wp$ function where $g_{2}$ and $g_{3}$ are constants which are determined by the periods. Then $\wp^{\prime}(x)=\sqrt{4 \wp(x)^{3}-g_{2} \wp(x)-g_{3}}$ and every elliptic function can be represented as a rational function of $\wp$ and $\wp^{\prime}$ (cf. [2]). In the case of Jacobi forms there are many representations for elliptic functions. For example, let $s n(x)=s n(x, k)$ where $k$ is a constant determined by the periods. Then

$$
s n^{\prime}(x)=c n(x) \cdot d n(x)=\sqrt{\left(1-s n^{2}(x)\right) \cdot\left(1-k^{2} s n^{2}(x)\right)}
$$

and every elliptic function can be represented as a rational function of $s n$ and $s n^{\prime}$. A similar statement holds for all the other 11 forms of the Jacobi elliptic functions (c.f. [2]).

Theorem 2.1 below gives the principal representations for our problems. The result follows directly from Singer [17] along with the isomorphism (via a change of variable from $x$ to $f$ ):

$$
\mathbb{K}(x, \sqrt{w(x)})\left[\frac{d}{d x}\right] \leftrightarrow \mathbb{K}\left(f, f^{\prime}\right)\left[\frac{d}{d f}\right]
$$

via

$$
x \mapsto f, \quad \sqrt{w(x)} \mapsto f^{\prime}, \quad \frac{d}{d x} \mapsto \frac{d}{d f}=\frac{1}{f^{\prime}} \frac{d}{d x}
$$

Theorem 2.1 (SINGER [17]). Let $L$ be a linear differential operator having coefficients in $\mathbb{K}\left(f, f^{\prime}\right)$ with $\mathbb{K}$ a field of constants and where $f$ satisfies $\left(f^{\prime}\right)^{2}=w(f)$ for some polynomial $w(z) \in \mathbb{K}[z]$. Then one has a decision procedure for finding solutions of $L(y)=0$ in $\mathbb{K}\left(f, f^{\prime}\right)$.

Thus suppose our coefficients are in Weierstrass form and that we are looking for solutions in the same form. Setting $f(x)=\wp\left(x ; g_{2}, g_{3}\right)^{1}$, the Weierstrass $\wp$ function, allows us to make use of the above formalism using $w(z)=4 z^{3}-g_{2} z-g_{3}$.

\footnotetext{
${ }^{1}$ As in classical texts, we drop the $g_{2}$ and $g_{3}$ arguments for $\wp$ in cases where this is obvious.
}

\subsection{Doubly-periodic Solutions of the Second Kind}

A second class of functions which are almost doubly periodic also plays an important role in the study of linear odes of the form (1). A function $F(x)$ is said to be doubly periodic of the second kind if there exist two periods $T, T^{\prime}$, and two constants $s, s^{\prime}$ such that

$$
F(x+T)=s \cdot F(x), \quad F\left(x+T^{\prime}\right)=s^{\prime} \cdot F(x)
$$

for all $x$ in the complex plane. The constants $s$ and $s^{\prime}$ are referred to as the period multipliers of $F(x)$. These functions are not, in general, truly periodic, as the value of $F(x)$ changes by a constant factor each time $x$ changes by the period.

Doubly periodic functions of the second kind are important from the following classical observation given by Picard (see Ince [13]) in 1879.

Theorem 2.2 (PICARD's THEOREM). If the coefficients of a homogeneous linear differential equation are doubly periodic functions of the independent variable, and all solutions of the equation are uniform (that is, path-independent), then the equation possesses at least one solution which is a doubly periodic function of the second kind.

We also note that when $F(x)$ is doubly periodic of the second kind then the ratio $G(x)=\frac{F^{\prime}(x)}{F(x)}$ is doubly periodic. For if $T$ and $T^{\prime}$ are the periods and $s, s^{\prime}$ the corresponding period multipliers of $F$ then

$$
G(x+T)=\frac{F^{\prime}(x+T)}{F(x+T)}=\frac{s F^{\prime}(x)}{s F(x)}=G(x)
$$

and similarly $G\left(x+T^{\prime}\right)=G(x)$. Thus the logarithmic derivative of any doubly periodic function of the second kind is doubly-periodic.

Conversely assume that $F(x)$ is a solution of our equation such that $\frac{F^{\prime}(x)}{F(x)}=G(x)$, a doubly periodic function. Then the logarithmic derivative of $\frac{F(x+T)}{F(x)}$ is $G(x+T)-G(x)$, which is zero by assumption. Hence there exists a constant $s$ such that $F(x+T)=s F(x)$. This also occurs for the second period $T^{\prime}$ and hence $F(x)$ would be doubly-periodic of the second kind. Thus finding doubly-periodic solutions of the second kind is equivalent to the search of first order doubly-periodic right factors of the associated linear differential operator.

We remark that, as mentioned earlier in the introduction, all functions are also assumed to not have any essential singularities. Hence the logarithmic derivative mentioned above will in fact be an elliptic function.

\subsection{Fundamental Bases}

To understand how doubly-periodic solutions of the second kind arise in the study of (1), one needs to first look at how fundamental bases behave at the periods. Let the set $\left\{f_{1}(x), f_{2}(x), \ldots, f_{n}(x)\right\}$ be a basis of solutions of (1) where all the coefficients are doubly periodic functions with periods $T, T^{\prime}$. Since the coefficients of (1) are doubly periodic we have that $f_{1}(x+T), f_{2}(x+T), \ldots, f_{n}(x+T)$, are also 
solutions of (1). Hence each can be expressed as a linear combination of the basis functions, that is,

$$
f_{j}(x+T)=a_{1 j} f_{1}(x)+a_{2 j} f_{2}(x)+\cdots+a_{n j} f_{n}(x)
$$

for some constants $a_{i j}$, independent of $x$. In matrix form this becomes

$$
\tilde{f}(x+T)=\tilde{f}(x) \cdot A
$$

where $\tilde{f}(x)=\left[f_{1}(x), \cdots, f_{n}(x)\right]$ and $A=\left[a_{i j}\right]_{n \times n}$. Similarly, we also have a constant matrix $B=\left[b_{i j}\right]$ which satisfies the matrix equation

$$
\tilde{f}\left(x+T^{\prime}\right)=\tilde{f}(x) \cdot B .
$$

The following gives some well known facts about the matrices $A$ and $B$ associated to a given fundamental system. We include a proof for completeness.

Theorem 2.3. Let $A$ and $B$ be constant matrices associated to a given fundamental basis $\left\{f_{1}(x), \ldots, f_{n}(x)\right\}$ of solutions of the linear ode (1). Then

(i) $A$ and $B$ are nonsingular

(ii) If $W(\tilde{f}(x))$ denotes the Wronskian of the fundamental system then

$$
\operatorname{det}(A)=\frac{W(\tilde{f}(x+T))}{W(\tilde{f}(x))} \text { and } \operatorname{det}(B)=\frac{W\left(\tilde{f}\left(x+T^{\prime}\right)\right)}{W(\tilde{f}(x))} \text {. }
$$

(iii) The products of the eigenvalues of $A$ and $B$, taking multiplicities into account, are

$$
\exp \left(-\int_{x}^{x+T} \frac{a_{n-1}(u)}{a_{n}(u)} d u\right) \text { and } \exp \left(-\int_{x}^{x+T^{\prime}} \frac{a_{n-1}(u)}{a_{n}(u)} d u\right),
$$
respectively.

(iv) Suppose $F(x)$ is a solution of (1) with

$$
F(x)=c_{1} \cdot f_{1}(x)+\cdots+c_{n} \cdot f_{n}(x) .
$$

Then $F(x)$ is doubly-periodic of the second kind with period multipliers $s$ and $s^{\prime}$ if and only if $s$ and $s^{\prime}$ are eigenvalues of $A$ and $B$, respectively, each having $\tilde{c}=$ $\left[c_{1}, \ldots, c_{n}\right]^{t}$ as a common eigenvector.

Proof: Differentiating both sides of (6) $n-1$ times with respect to $x$ gives $n-1$ additional equations of the form

$$
\tilde{f}^{\prime}(x+T)=\tilde{f}^{\prime}(x) \cdot A, \ldots, \tilde{f}^{(n-1)}(x+T)=\tilde{f}^{(n-1)}(x) \cdot A .
$$

Combining these into a single matrix equation, we have

$$
\begin{aligned}
& {\left[\begin{array}{lll|l}
\tilde{f}(x+T) & & \cdots & \tilde{f}^{(n-1)}(x+T)
\end{array}\right]^{t}} \\
& =\left[\begin{array}{lll|l}
\tilde{f}(x) & \mid & \cdots & \tilde{f}^{(n-1)}(x)
\end{array}\right]^{t} \cdot A .
\end{aligned}
$$

Since we have a fundamental system the Wronskian matrix $\left[\begin{array}{l|l|l|}\tilde{f}(x) & \cdots & \tilde{f}^{(n-1)}(x)\end{array}\right]^{t}$ is nonsingular and hence so is $A$. A similar argument shows that $B$ is also nonsingular hence we have (i). Part (ii) follows by taking determinants of both sides of (9). Part (iii) follows from (ii) along with Abel's identity [13, p. 75].

Suppose now that $F(x)$ is a solution of (1) with

$$
F(x)=c_{1} f_{1}(x)+\cdots+c_{n} f_{n}(x)=\tilde{f}(x) \cdot \tilde{c}
$$

where $\tilde{c}=\left[c_{1}, \ldots, c_{n}\right]^{t}$. Then

$$
F(x+T)=\tilde{f}(x+T) \cdot c=\tilde{f}(x) \cdot A \cdot \tilde{c}
$$

and

$$
F\left(x+T^{\prime}\right)=\tilde{f}\left(x+T^{\prime}\right) \cdot c=\tilde{f}(x) \cdot B \cdot \tilde{c} .
$$

Notice that for any constants $s$ and $s^{\prime}$ equations (11) and (12) imply that

$$
s F(x)=F(x+T) \Longleftrightarrow s \tilde{f}(x) \cdot \tilde{c}=\tilde{f}(x) \cdot A \cdot \tilde{c}
$$

and

$$
s^{\prime} F(x)=F\left(x+T^{\prime}\right) \Longleftrightarrow s^{\prime} \tilde{f}(x) \cdot \tilde{c}=\tilde{f}(x) \cdot B \cdot \tilde{c} .
$$

Using a similar argument as before we see that equations (13) and (14) are equivalent to

$$
s \cdot \tilde{c}=A \cdot \tilde{c} \text { and } s^{\prime} \cdot \tilde{c}=B \cdot \tilde{c}
$$

so that $s F(x)=F(x+T)$ and $s^{\prime} F(x)=F\left(x+T^{\prime}\right)$ if and only if $s$ and $s^{\prime}$ are in fact eigenvalues of the matrices $A$ and $B$, respectively, having a common eigenvector $\tilde{c}$.

Remark 2.4. The conditions of Picard's Theorem require that all solutions of (1) be path-independent. As such one can traverse a path from $x$ to $x+T$ and then to $x+T+T^{\prime}$ and expect to end at the same place as if one has traversed a path from $x$ to $x+T^{\prime}$ to $x+T^{\prime}+T$. Using equations (6) and (7) implies that $A$ and $B$ therefore commute. An elementary result from linear algebra [8] implies that these matrices then have eigenvalues $s$ and $s^{\prime}$ with a common eigenvector. Picard's Theorem thus follows from part (iv) of Theorem 2.3.

Remark 2.5. The multipliers are eigenvalues and thus independent of the fundamental system.

Remark 2.6. Once one doubly periodic solution of the second kind has been found we may use reduction of order to find additional solutions. In the present case, reduction of order for (1) results in a new ode also having doubly periodic coefficients, and all solutions uniform. Picard's Theorem therefore implies that this new ode also has at least one solution which is doubly periodic of the second kind. This may be repeated, to show that there is a basis of solutions of (1) of the form

$$
\begin{gathered}
\left\{y_{1}(x)=\phi_{1}(x), \quad y_{2}(x)=\phi_{1}(x) \int \phi_{2}(x) d x,\right. \\
\left.\ldots, \quad y_{n}(x)=\phi_{1}(x) \int \phi_{2}(x) \cdots \int \phi_{n}(x)(d x)^{n-1}\right\},
\end{gathered}
$$

where the $\phi_{i}(x)$ are doubly-periodic of the second kind (Ince [13, p. 376]).

\section{SOLVING 2ND ORDER EQUATIONS VIA SYMMETRIC POWERS}

In this section we are interested in determining when a second order linear ode (1) has a basis of solutions which are doubly-periodic of the second kind. From the comments at the end of section 2.2 we know that this becomes equivalent to searching for elliptic function first order factors of the associated linear differential operator. Thus, in the case of second order equations, we can look to apply the same 
techniques as used in modern versions of Kovacic's algorithm [18]. In particular, we can reduce our problem to finding elliptic function solutions of a related third order linear ode.

It is well known that using the transform

$$
y(x)=\exp \left(-\int \frac{a_{n-1}(x)}{n a_{n}(x)} d x\right) \cdot u(x)
$$

converts an $n$-th order ode in $y(x)$ into an ode in $u(x)$ having the second highest term 0 . In the case of second order equations this converts

$$
a_{2}(x) \cdot y^{\prime \prime}(x)+a_{1}(x) \cdot y^{\prime}(x)+a_{0}(x) \cdot y(x)=0
$$

into

$$
u^{\prime \prime}(x)-r(x) \cdot u(x)=0
$$

with $r(x)=\left(\frac{a_{1}(x)}{2 a_{2}(x)}\right)^{\prime}+\left(\frac{a_{1}(x)}{2 a_{2}(x)}\right)^{2}-\frac{a_{0}(x)}{a_{2}(x)}$.

The following is central to the results in this section.

Lemma 3.1. Assume that equation (1) has coefficient $a_{n-1}(x)=0$ and that $\left\{y_{1}(x), \ldots, y_{n}(x)\right\}$ is a basis of solutions with each $y_{i}(x)$ doubly periodic of the second kind. Then $z(x)=y_{1}(x) \cdots y_{n}(x)$ is doubly periodic.

Proof: $\quad$ Let $T$ and $T^{\prime}$ be the periods for the coefficients of the linear ode and suppose that $y_{i}(x+T)=s_{i} \cdot y_{i}(x)$, $y_{i}\left(x+T^{\prime}\right)=s_{i}^{\prime} \cdot y_{i}(x)$, for $i=1, \ldots, n$. Then from part (iii) of Theorem 2.3 we have that $s_{1} \cdots s_{n}=1$. Therefore

$$
\begin{aligned}
z(x+T) & =y_{1}(x+T) \cdots y_{n}(x+T) \\
& =\left(s_{1} \cdots s_{n}\right) y_{1}(x) \cdots y_{n}(x) \\
& =z(x) .
\end{aligned}
$$

Similarly, $z\left(x+T^{\prime}\right)=z(x)$ for the second period $T^{\prime}$ and hence $z(x)$ is doubly periodic.

Consider first the case of finding a basis of solutions of a second order linear ode of the form (17). We show in Corollary 3.2 and Theorem 3.3 that solutions of such an equation can be determined by looking for special solutions of

$$
y^{\prime \prime \prime}(x)-4 \cdot r(x) \cdot y^{\prime}(x)-2 \cdot r^{\prime}(x) \cdot y(x)=0,
$$

the second symmetric power of equation (17).

Corollary 3.2. Suppose that equation (17) has a basis of solutions which are doubly-periodic of the second kind. Then the second symmetric power (19) has at least one solution $z(x)$ which is doubly periodic.

Proof: It is well known (c.f. [14]) that a basis for solutions of equation (19) is given by $\left\{y_{1}(x)^{2}, y_{1}(x) y_{2}(x), y_{2}(x)^{2}\right\}$ where $\left\{y_{1}(x), y_{2}(x)\right\}$ is a basis for equation (17). The corollary thus follows directly from Lemma 3.1.

The following theorem shows how to build a basis for (17) from a solution of (19).

Theorem 3.3. Let $z(x)$ be a solution of (19) and set

$$
y_{1}(x)=\sqrt{z(x)} \cdot \exp \left(-\frac{C}{2} \int \frac{d x}{z(x)}\right)
$$

and

$$
y_{2}(x)=\sqrt{z(x)} \cdot \exp \left(\frac{C}{2} \int \frac{d x}{z(x)}\right)
$$

where $C$ is a constant given by

$$
C^{2}=z^{\prime}(x)^{2}-2 \cdot z(x) \cdot z^{\prime \prime}(x)+4 \cdot r(x) \cdot z(x)^{2} .
$$

If $C \neq 0$, then $y_{1}(x), y_{2}(x)$ are linearly independent and form a basis of (17). If $C=0$, then a basis for the solution space of (17) is given by $y_{1}(x)$ and $y_{2}(x)=\sqrt{z(x)} \int \frac{1}{z(x)} d x$.

Proof: $\quad$ Let $z(x)=y_{1}(x) \cdot y_{2}(x)$ and $C$ be the Wronskian of $y_{1}(x), y_{2}(x)$. Note that $C$ is a constant by Abel's identity [13]. From $z^{\prime}(x)=y_{1}^{\prime}(x) \cdot y_{2}(x)+y_{1}(x) \cdot y_{2}^{\prime}(x)$ we have that

$$
\frac{z^{\prime}(x)}{z(x)}=\frac{y_{1}^{\prime}(x)}{y_{1}(x)}+\frac{y_{2}^{\prime}(x)}{y_{2}(x)}
$$

while $C=y_{2}^{\prime}(x) \cdot y_{1}(x)-y_{2}(x) \cdot y_{1}^{\prime}(x)$ implies that

$$
\frac{C}{z(x)}=\frac{y_{2}^{\prime}(x)}{y_{2}(x)}-\frac{y_{1}^{\prime}(x)}{y_{1}(x)}
$$

Taking equations (23) and (24) together gives

$$
\frac{y_{1}^{\prime}(x)}{y_{1}(x)}=\frac{z^{\prime}(x)-C}{2 \cdot z(x)} \text { and } \frac{y_{2}^{\prime}(x)}{y_{2}(x)}=\frac{z^{\prime}(x)+C}{2 \cdot z(x)}
$$

which gives (21). In addition, differentiating $\frac{y_{1}^{\prime}(x)}{y_{1}(x)}$ gives

$\frac{y_{1}^{\prime \prime}(x)}{y_{1}(x)}-\left(\frac{y_{1}^{\prime}(x)}{y_{1}(x)}\right)^{2}=\frac{2 \cdot z(x) \cdot z^{\prime \prime}(x)-2 \cdot z^{\prime}(x)^{2}+2 \cdot C \cdot z^{\prime}(x)}{4 \cdot z(x)^{2}}$

so that

$$
\frac{y_{1}^{\prime \prime}(x)}{y_{1}(x)}=\frac{2 \cdot z(x) \cdot z^{\prime \prime}(x)-z^{\prime}(x)^{2}+C^{2}}{4 \cdot z(x)^{2}} .
$$

Since $\frac{y_{1}^{\prime \prime}(x)}{y_{1}(x)}=r(x)$ we get identity (22). Now, since $C$ is the Wronskian of $y_{1}(x), y_{2}(x), C \neq 0$ implies that $y_{1}(x)$, $y_{2}(x)$ are linearly independent and form a basis for (17). On the other hand, if $C=0$, the formulas (21) become identical, with one solution $y_{1}(x)=\sqrt{z(x)}$. A second solution, from standard reduction of order techniques, is then $y_{2}(x)=y_{1}(x) \int \frac{1}{y_{1}^{2}(x)} d x=\sqrt{z(x)} \int \frac{1}{z(x)} d x$.

Remark 3.4. Theorem 3.3 appears to have been known in the 1800's by Hermite [11], Brioschi [5], and Halphen [10], at least in the case of Lamé's equation. Brioschi also used the result to analyze Mathieu's equation

$$
y^{\prime \prime}(x)+\left(p-k^{2} \cdot v^{2} \cdot \cos ^{2}(x)\right) \cdot y(x)=0
$$

(so $r(x)=k^{2} v^{2} \cos ^{2}(x)-p$ ) where the coefficients are only periodic. Indeed, in this case equation (22) is known as Brioschi's Identity.

In the case of second order linear odes having elliptic function coefficients, Theorem 3.3 provides a simple method for finding a general solution for the ode whenever two independent solutions, doubly periodic of the second kind, exist. Classically these solutions are given in terms of the Weierstrass Zeta and Sigma functions which are defined in terms of $\wp$ via

$$
\zeta^{\prime}(x)=-\wp(x) \text { and } \frac{\sigma^{\prime}(x)}{\sigma(x)}=\zeta(x) .
$$

These Weierstrass functions are not periodic but rather quasiperiodic (cf. [1]).

Example 3.5. Consider Lamé's equation (2), with $n$ any positive integer, $B, g_{2}$, and $g_{3}$ arbitrary constants. We 
search for solutions that are doubly periodic of the second kind. The corresponding symmetric power is given by

$$
z^{\prime \prime \prime}(x)-4(n(n+1) \wp(x)+B) z^{\prime}(x)-2 n(n+1) \wp^{\prime}(x) z(x)=0
$$

and this has a doubly periodic solution. Thus we can compute solutions of (2) which are doubly periodic of the second kind.

We can illustrate with some examples for $n$ small. For example, if $n=1$, then solutions $z(x)$ of (27) which are rational in $\wp$ and $\wp^{\prime}$ are given by multiples of $\{\wp-B\}$. Then $C^{2}=z^{\prime}(x)^{2}-2 z(x) z^{\prime \prime}(x)+4 r(x) z(x)^{2}=4 B^{3}-g_{2} B-g_{3}$, which is a constant. If $C \neq 0$, two independent solutions are

$$
\begin{aligned}
\tilde{y_{1}}(x) & =\exp \left(\int \frac{z_{1}^{\prime}(x)-C}{2 z_{1}(x)} d x\right) \\
& =\exp \left(\frac{1}{2} \int \frac{\wp^{\prime}(x)-\sqrt{4 B^{3}-g_{2} B-g_{3}}}{\wp(x)-B} d x\right) \\
\tilde{y_{2}}(x) & =\exp \left(\int \frac{z_{1}^{\prime}(x)+C}{2 z_{1}(x)} d x\right) \\
& =\exp \left(\frac{1}{2} \int \frac{\wp^{\prime}(x)+\sqrt{4 B^{3}-g_{2} B-g_{3}}}{\wp(x)-B} d x\right) .
\end{aligned}
$$

We can also return the final solution in a form found in standard texts. Indeed, up to an additive constant, we have

$\frac{1}{2} \int \frac{\wp^{\prime}(x)-\sqrt{4 B^{3}-g_{2} B-g_{3}}}{\wp(x)-B} d x=\ln \left(\frac{\sigma(x+a)}{\sigma(x) \sigma(a)}\right)-x \zeta(a)$

and

$\frac{1}{2} \int \frac{\wp^{\prime}(x)+\sqrt{4 B^{3}-g_{2} B-g_{3}}}{\wp(x)-B} d x=\ln \left(\frac{\sigma(x+a)}{\sigma(x) \sigma(a)}\right)+x \zeta(a)$

where $a$ is a constant such that $\wp(a)=B(c f .[1,2])$. Hence, from (28), we have the solutions

$$
\begin{aligned}
& y_{1}(x)=\exp \left(\ln \left(\frac{\sigma(x+a)}{\sigma(x) \sigma(a)}\right)-x \zeta(a)\right)=\frac{\sigma(x+a)}{\sigma(x) \sigma(a)} e^{-x \zeta(a)} \\
& y_{2}(x)=\frac{\sigma(x-a)}{\sigma(x) \sigma(a)} e^{x \zeta(a)}
\end{aligned}
$$

where $a$ is such that $\wp(a)=B$.

For higher values of $n$, solutions for $z(x)$ and $C$, and hence for $y_{1}(x)$ and $y_{2}(x)$, are still obtained. However, it becomes more difficult to evaluate the integrals for increasing values of $n$ so we just give $z(x)$ and $C^{2}$ for $n=2$ and 3 :

$$
\begin{aligned}
n=2: z(x)= & -\frac{1}{4} g_{2}+\frac{1}{9} B^{2}-\frac{1}{3} B \wp(x)+\wp(x)^{2} \\
C^{2}= & -g_{2} g_{3}+\frac{1}{3} g_{2}^{2} B+\frac{1}{3} g_{3} B^{2} \\
& -\frac{7}{27} g_{2} B^{3}+\frac{4}{81} B^{5} \\
n=3: z(x)= & -\frac{1}{4} g_{3}+\frac{1}{15} g_{2} B-\frac{1}{225} B^{3}-\frac{1}{4} g_{2} \wp(x) \\
& +\frac{2}{75} B^{2} \wp(x)-\frac{1}{5} B \wp(x)^{2}+\wp(x)^{3} \\
C^{2}= & -\frac{1}{60} g_{2}^{3} B+\frac{9}{20} g_{3}^{2} B-\frac{9}{50} g_{2} g_{3} B^{2} \\
& +\frac{31}{1500} g_{2}^{2} B^{3}+\frac{22}{1875} g_{3} B^{4} \\
& -\frac{14}{5625} g_{2} B^{5}+\frac{4}{50625} B^{7}
\end{aligned}
$$

Using the computer algebra system Maple, the solutions $y_{1}(x), y_{2}(x)$ generated from these solutions for $z(x)$ and $C$ have all been verified to be solutions of the corresponding instance of Lamé's equation.

\section{SOLVING 2ND ORDER EQUATIONS VIA DIFFERENTIAL FACTORIZATION}

If (17) has a doubly periodic of the second kind solution, then by Theorem 3.3 such a solution can be found when there is a doubly periodic solution of (19). However, if (19) has no doubly periodic solutions, then this does not necessarily imply that there are no doubly periodic solutions of the second kind for (17). For example, the linear ode

$$
y^{\prime \prime}(x)-\left(\wp(x)^{2}-\wp^{\prime}(x)\right) y(x)=0
$$

has a solution which is doubly periodic of the second kind even though its second symmetric power has no doubly periodic solutions. In this section we give an alternate method which will solve such equations.

Recall from subsection 2.2 that the logarithmic derivative of any doubly periodic function of the second kind is doubly-periodic. As such any doubly-periodic solution of the second kind of (1) gives a first order right factor of our original equation. Finding first order factors of a linear operator is equivalent to finding exponential solutions of the corresponding linear ode. In the case of linear differential operators in the domain $\mathbb{K}(x)\left[D_{x}\right]$ where $D_{x}=\frac{d}{d x}$ we can find exponential solutions with existing algorithms such as $[4,6,12]$ while in our domain we can find exponential solutions via the method from [17].

Assume now that our linear ode (1) has order 2. By using a reduction of the form (15) we can reduce the problem of finding doubly-periodic solutions of the second kind to finding right hand factors of

$$
L=D_{x}^{2}-r(x) \text { where } r(x)=a(\wp(x))+b(\wp(x)) \wp^{\prime}(x)
$$

with $a(z), b(z) \in \mathbb{K}(z)$.

Notice that if one does a substitution of the form $z=\wp(x)$ then $D_{x}=\sqrt{w(z)} D_{z}$ with $D_{z}=\frac{d}{d z}$ where $w(z) \in \mathbb{K}[z]$ satisfies $\wp^{\prime}(x)^{2}=w(\wp(x))$. Thus we can express $L$ as a differential operator in $\mathbb{K}(z, \sqrt{w(z)})\left[D_{z}\right]$ by

$$
L=w(z) D_{z}^{2}+\frac{w^{\prime}(z)}{2} D_{z}-a(z)-b(z) \sqrt{w(z)} .
$$

We may assume that $b(z) \neq 0$ (otherwise $L$ is in $\mathbb{K}(z)\left[D_{z}\right]$ in which case we can use the Kovacic algorithm instead of the method given below). We can create a fourth order operator having rational coefficients by

$$
\hat{L}:=\operatorname{symmetric\_ product}(L, \bar{L}) \in \mathbb{K}(z)\left[D_{z}\right]
$$

where $\bar{L} \in \mathbb{K}(z, \sqrt{w(z)})\left[D_{z}\right]$ is the conjugate of $L$ (that is, every occurrence of $\sqrt{w(z)}$ is replaced by $-\sqrt{w(z)})$. The symmetric product produces an operator $\hat{L}$ of minimal order such that the product of any solution of $L$ and any solution of $\bar{L}$ is a solution of $\hat{L}$. 


\section{Theorem 4.1.}

(a) Suppose $D_{x}-s(x)$ is a right factor of $L$ with

$$
s(x)=u(\wp(x))+v(\wp(x)) \wp^{\prime}(x) .
$$

Then $D_{z}-2 v(z)$ is a right factor of $\hat{L}$.

(b) Suppose $D_{z}-c(z)$ is a right factor of $\hat{L}$. Set

$$
\begin{aligned}
v(z) & =\frac{c(z)}{2} \\
t(z) & =v(z)^{2} w(z)+v^{\prime}(z) w(z)+\frac{1}{2} v(z) w^{\prime}(z), \\
u(z) & =\frac{1}{2 b(z)}\left(a^{\prime}(z)+4 a(z) v(z)-4 t(z) v(z)-t^{\prime}(z)\right) .
\end{aligned}
$$

If $a(z)=u(z)^{2}+t(z)$, then we have the factorization

$$
L=\left(D_{x}+s(x)\right)\left(D_{x}-s(x)\right)
$$

where $s(x)=u(\wp(x))+v(\wp(x)) \wp^{\prime}(x)$.

Proof: Suppose that $D_{x}-s(x)$ is a right factor of $L$. Changing coordinates of this factor via $z=\wp(x)$ and simplifying ensures that $D_{z}-\left(v(z)+\frac{u(z)}{w(z)} \sqrt{w(z)}\right)$ is a right factor of $L$. As such $D_{z}-\left(v(z)-\frac{u(z)}{w(z)} \sqrt{w(z)}\right)$ is a right factor of $\bar{L}$ and so $D_{z}-2 v(z)$ is a right factor of $\hat{L}$, the symmetric product of $L$ and $\bar{L}$. Thus for each right factor of $\hat{L}$ we obtain the corresponding formula for $v(z)$.

In order to obtain a formula for the component $u(z)$ first notice that $D_{x}^{2}-r(x)=\left(D_{x}+s(x)\right)\left(D_{x}-s(x)\right)$ if and only if

$$
r(x)=s^{\prime}(x)+s(x)^{2}
$$

which in turn occurs if and only if

$$
\begin{aligned}
& a(z)=u(z)^{2}+v(z)^{2} w(z)+v^{\prime}(z) w(z)+\frac{1}{2} v(z) w^{\prime}(z) \\
& b(z)=u^{\prime}(z)+2 u(z) v(z) .
\end{aligned}
$$

Taking derivatives of equation (37), multiplying equation (38) by $2 u(z)$, taking differences of the two resulting equations and then substituting for $u(z)^{2}$ using equation (37) gives the formula for $u(z)$.

Example 4.2. Consider first equation (32) from the start of this section. In this case the symmetric product has a right factor of the form $D_{z}$. As such both $v(z)=0$ and $t(z)=0$. Further computation gives $u(z)=-z$ and hence the linear ode associated to (32) has a right factor $D_{x}+\wp(x)$. This in turn gives a doubly-periodic of the second kind solution of (32) of the form

$$
e^{-\int \wp(x) d x}=e^{\zeta(x)}
$$

Example 4.3. Consider now the equation

$$
y^{\prime \prime}(x)-\left(6 \wp(x)+1-\frac{g_{2}}{2 \wp(x)}+\frac{2}{\wp(x)} \wp^{\prime}(x)\right) y(x)=0 .
$$

As before, the corresponding symmetric power equation (17) does not have a doubly-periodic solution. Forming the operators $L$ and $\bar{L}$, taking the symmetric product $\hat{L}$ and factoring then gives a right factor of the form $D_{z}-\frac{2}{z}$. Using Theorem 4.1 we determine that $v(z)=\frac{1}{z}$ and $u(z)=1$. Hence $D_{x}-\left(1+\frac{\wp^{\prime}(x)}{\wp(x)}\right)$ is a right factor of our original second order operator. A doubly-periodic solution of the second kind for our example is then given by

$$
e^{\int\left(1+\frac{\wp^{\prime}(x)}{\wp(x)}\right) d x}=e^{x} \wp(x) .
$$

For both examples, a second independent solution for the ode can be found using reduction of order.

Remark 4.4. We can obtain a similar result for factorizations of the form

$$
L=D_{z}^{2}-r(z)=\left(D_{z}+s(z)\right)\left(D_{z}-s(z)\right)
$$

where $r(z)=a(z)+b(z) \sqrt{w(z)}$ and $s(z)=u(z)+v(z) \sqrt{w(z)}$. Indeed, if $D_{z}-c(z)$ is a right factor of $L \in \mathbb{K}(z)\left[D_{z}\right]$ then the conditions for such a factorization would be $u(z)=\frac{c(z)}{2}$ and

$$
v(z)=\frac{\left(a^{\prime}(z)+4 u(z) a(z)-4 u(z)^{3}-6 u(z) u^{\prime}(z)-u^{\prime \prime}(z)\right)}{2 b(z) w(z)}
$$

with $a(z)=u(z)^{2}+v(z)^{2} w(z)+u^{\prime}(z)$. Thus one can obtain a similar procedure by first changing coordinate systems, then normalizing to remove the linear term of the operator in $\mathbb{K}(x, \sqrt{w(z)})\left[D_{z}\right]$ and finally finding right factors of the resulting symmetric product in $\mathbb{K}(z)\left[D_{z}\right]$.

Remark 4.5 (COMPleteness). Theorem 4.1 provides a complete method for finding doubly periodic solutions of the second kind for a second order linear operator of the form (34). Namely, compute all exponential solutions of $\hat{L}$ which in turn gives all first order right factors $D_{z}-c(z)$ of $\hat{L}$. Then search for those $c(z)$, if any, for which the condition $a(z)=$ $u(z)^{2}+t(z)$ in Theorem 4.1 holds. Those then produce the factorization (36).

Remark 4.6 (IMPLEMENTATION). An algorithm for finding all solutions doubly periodic of the second kind for second order equations has been implemented and will be in the coming version of Maple. In implementing our results [7] we have taken advantage of the existence of an efficient implementation of the method from section 3. By first trying the methods from section 3 we do not need to implement the complete version of Theorem 4.1. We mention two reasons here. First, because we have tried the method of symmetric powers, we do not need to compute all exponential solutions of $\hat{L}$. Instead we only search for those that are relatively easy to compute, namely those that do not involve algebraic extensions of the constants, which makes our implementation much faster. Secondly, if there exist infinitely many first order right factors $D_{z}-c(z)$ of $\hat{L}$, then all such $c(z)$ can be represented by finitely many $c(z)$ that contain parameters, and we have to find the parameter values for which $a(z)=u(z)^{2}+t(z)$. While finding these parameters is not hard to implement, we have not done so.

We can justify the simplifications in our implementation of Theorem 4.1 as follows. If we have two first order factors, then the method of section 3 will find them. Hence we only need to worry about the case where we have a unique first order factor. Since this factor does not require an extension of the field of constants, it is also the case that the factor $D_{z}-c(z)$ of $\hat{L}$ does not require extensions of the field of constants. Hence no solutions are in fact lost by not extending our coefficient field. Similarly, suppose $\hat{L}$ has infinitely many right factors. In this case, where $L$ has a unique first order right factor while $\hat{L}$ has infinitely many, 
it can be shown that the second symmetric power will have an elliptic function solution, and hence the method from section 3 will again work. Thus, our simplified implementation for Theorem 4.1 combined with the method in section 3 gives a complete algorithm for solutions which are doubly periodic of the second kind for second order linear odes with elliptic function coefficients.

\section{CONCLUSION}

We have implemented an efficient algorithm for finding elliptic function solutions of arbitrary order linear odes of the form (1) along with a procedure based on the results of section 3 to find general solutions for (1) of the second order. The algorithms are included in version 9 of the Maple computer algebra system, used both inside dsolve, the differential equation solver, and as a stand alone function $d p e-$ riodic_sols in the DEtools package. The extensions reported in section 4 have been added and will be included in the next version of Maple. A report on these implementations will be forthcoming [7].

There are a number of topics for future research. We are interested in developing efficient and complete methods for all solutions which are doubly-periodic of the second kind, for odes of the form (1) which have orders higher than 2. In addition, we are interested in finding differential factorizations of higher order linear differential operators in $\mathbb{K}[z, \sqrt{w(z)}]$ with $w(z) \in \mathbb{K}[z]$. Finally, in the case of second order odes we are interested in extending the techniques used in this paper to compute general Liouvillian solutions for (1) when there are no solutions doubly periodic of the second kind.

Acknowledgements: We would like to thank Anne Fredet and the anonymous referees for their helpful comments on this paper.

\section{REFERENCES}

[1] M. Abramowitz and I.A. Stegun, Handbook of Mathematical Functions, Dover Publications, New York, (1972).

[2] N.I. Akhiezer, Elements of the Theory of Elliptic Functions, American Mathematical Society, (1990).

[3] P.E. Appell and E. Lacour, Principes de la Théorie des Fonctions Elliptiques et Applications, Gauthier-Villars et Cie, (1897).
[4] E. Beke, Die Irreduzibilität der homogenen linearen Differentialgleichungen, Math. Ann. 45, p. 278-294, (1894).

[5] F. Brioschi, Sur l'equation de Lamé. Comptes Rendus de l'Academie des Sciences, 86, 313-315, (1878).

[6] M. Bronstein, Linear Ordinary Differential Equations: breaking through the order 2 barrier, Proceedings of ISSAC'92 (P. Wang ed.), 42-48, (1992).

[7] R.F. Burger, G. Labahn and M. van Hoeij, An algorithm to solve linear odes with elliptic function coefficients, Manuscript.

[8] W.L. Ferrar, Algebra; A Text-book of Determinants, Matrices, and Algebraic Forms, 2nd edition, Oxford University Press, (1957).

[9] A.R. Forsyth, Differential Equations I-VI, Cambridge University Press, Cambridge, England, (1906).

[10] G.H. Halphen, Traité des Fonctions Elliptiques et de Leurs Applications, Volume 2. Gauthier-Villars et Fils, (1888).

[11] C. Hermite, Oeuvres, Volume 3. Gauthier-Villars, (1912).

[12] M. van Hoeij, Factorization of Differential Operators with Rational Function Coefficients, Journal of Symbolic Computation 24 537-561 (1997).

[13] E. Ince, Ordinary Differential Equations, Dover Publications, New York, (1956).

[14] E. Kamke, Differentialgleichungen: Lösungsmethoden und Lösungen, Chelsea Publishing Co, New York, (1959).

[15] E. Picard, Sur une généralisation des fonctions périodiques et sur certaines équations différentielles linéaires, Comptes Rendus de l'Academie des Sciences, 89, 140-145 (1879).

[16] M.F. Singer, Liouvillian Solutions of n-th order Homogeneous Linear Differential Equations, American Journal of Mathematics, 103 661-682 (1981).

[17] M.F. Singer, Liouvillian Solutions of Linear Differential Equations with Liouvillian Coefficients, Journal of Symbolic Computation, 11 251-273 (1991).

[18] F. Ulmer and J.A. Weil, A Note on Kovacic's Algorithm, Journal of Symbolic Computation, 22 179-200 (1996). 